$63^{\text {ème }}$ Congrès de la SFCO, 03025 (2015)

DOI:10.1051/sfco/20156303025

(C) Owned by the authors, published by EDP Sciences, 2015

\title{
Simulation des reconstructions implantaires sur lambeaux de fibula : évaluation de l'utilisation des guides chirurgicaux
}

\author{
Jalbert $\mathrm{F}^{* * * *}$, Alpy $\mathrm{A}^{*}$, Fenelon $\mathrm{M}^{*}$, Lhermusier $\mathrm{E}^{*}$, Pronost $\mathrm{X}^{* *}$, Lopez $\mathrm{R}^{*}$, Boutault $\mathrm{F}^{*}$ \\ * CHU Toulouse, 1 place Dr. Baylac, 31000 Toulouse \\ ** Clinique Pasteur, 45 avenue de Lombez, 31300 Toulouse
}

La reconstruction des larges défects osseux maxillaires et mandibulaires fait souvent appelà une reconstruction par lambeau libre micro-anastomosé osseux, comme le lambeau de fibula. L’objectif de la reconstruction est ici double, tant morphologique que fonctionnel. La restauration fonctionnelle, et donc prothétique, est désormais facilitée par la mise en place d'implants dentaires à ancrage osseux sur la fibula. L'insertion de ces implants, la conformation spatiale du lambeau et même la prothèse peuvent être simulés de manière tridimensionnelle afin d'aboutir à une réhabilitation optimale. Les guides chirurgicaux sont de précieux outils pour transposer cette planification sur le site de la reconstruction dans ces réhabilitations complexes, ils nous servent dans notre expérience, à la fois de guide de coupe de la fibula, et de guide implantaire.

Nous avons évalué tout d'abord la fiabilité du guide chirurgical utilisé (PositGuide ${ }^{\circledR}$ ) en comparant la simulation de l'axe et du positionnement des implants au résultat post opératoire. Une étude qualitative et fonctionnelle a ensuite également été menée en évaluant le retentissement sur la qualité de vie de nos patients.

Nous avons évalué 9 patients ayant bénéficiés de cette méthodologie de reconstruction. L'étude a porté sur 58 implants. Tous nos patients présentaient des défects osseuxmaxillaires ou mandibulaires d'étiologies diverses (traumatisme balistique, séquelles d'exérèses carcinologiques, ...) nécessitant une reconstruction par lambeau de fibula. Les résultats montrent une précision du positionnement implantaire tout à fait satisfaisante comparativement à la simulation. Cela nous a même autorisé à réaliserplusieurs cas « d’implantation - mise en charge immédiate » dans ces indications.

Si la place du lambeau libre de fibula dans la reconstruction maxillo-faciale n'est plus à discuter, les guides chirurgicaux que nous utilisons doivent être précis afin de nous permettre d'élargir les indications implantaires à des situations anatomiques complexes. En métal, peu encombrant, pouvant intégrer un guide de coupe de fibula, le Positguide ${ }^{\circledR}$ apparaît particulièrement intéressant dans cette indication.

Microsurgical reconstruction of the jawwithfibulargrafts and implants. Raoul $G$ et al., $J$ CraniofacSurg. 2009 Nov;20(6):2105-17

[Dental implants and microvascular free fibulaflap: 23 patients]. Bodard et al. RevStomatolChirMaxillofac. 2008 Dec;109(6):363-6

Dental implant placement aftermandibular reconstruction by microvascular free fibulaflap: currentknowledge and remaining questions. Anne-Gaëlle B et al., Oral Oncol. 2011 Dec;47(12):1099104

This is an Open Access article distributed under the terms of the Creative Commons Attribution License 4.0, which permits unrestricted use, distribution, and reproduction in any medium, provided the original work is properly cited. 
$63^{\text {ème }}$ Congrès de la SFCO

Nom et adresse du conférencier

Florian JALBERT

CHU Toulouse

1 place Dr. Baylac

31000 Toulouse (France)

f.jalbert@orange.fr 